Die nachfolgenden Empfehlungen wurden aus der Sicht der Strafverteidigung im Kanton Zürich verfasst. In anderen Kantonen gelten zum Teil leicht abweichende Verfahren und Begriffe. Zudem ist das Strafuntersuchungsklima von Kanton zu Kanton verschieden, beispielsweise in der Frage, wann die Strafuntersuchungsbehörde Zwangsmassnahmen wie eine Hausdurchsuchung oder Untersuchungshaft anordnet. Grundlegende Empfehlungen des Beitrags gelten hingegen überall.*

\section{Der betroffene Arzt soll}

- aus Rücksicht auf das Patientengeheimnis die Siegelung der Krankengeschichte verlangen,

- nur Aussagen machen, wenn der Vorwurf präzise und klar formuliert wird, und selbst dann Aussagen kurz und knapp halten.

Hanspeter Kuhn, Fürsprecher stv. Generalsekretär FMH

\title{
Strafuntersuchung gegen Ärzte - wie sollen sich Betroffene verhalten?
}

Tanja Knodel

\footnotetext{
* Ein Dank für die ergänzende Durchsicht geht an Fürsprecher Fredi Hänni, Bern. HPK
}

\section{Einleitung}

In letzter Zeit mehren sich Strafuntersuchungen gegen Ärzte. Diese geraten beispielsweise unter Beschuss, wenn in arbeitsrechtlichen Verfahren ein Arztzeugnis darüber entscheidet, ob ein Arbeitsverhältnis gekündigt werden kann oder ob eine Lohnfortzahlungspflicht besteht. Oftmals wird versucht, an der Glaubwürdigkeit eines Arztzeugnisses zu rütteln, indem eine Strafanzeige gegen den ausstellenden Arzt eingereicht wird. Wer ein falsches ärztliches Zeugnis ausstellt, erfüllt den Tatbestand des Art. 318 StGB und wird mit Geldstrafe oder Freiheitsstrafe bis zu 3 Jahren bestraft, bei fahrlässiger Begehung mit Busse. Ein Arzt läuft bei seiner Tätigkeit aber auch Gefahr, weitere Tatbestände des Schweizerischen Strafgesetzbuches zu erfüllen, z.B. die Erstellung eines falschen Gutachtens gemäss Art. 308 StGB oder die Verletzung des Berufsgeheimnisses gemäss Art. 321 StGB. Schliesslich bleibt der Arzt auch nicht vom Vorwurf begangener Gewaltdelikte, wie z. B. fahrlässiger Körperverletzung (Art. 125 StGB), fahrlässiger Tötung (Art. 117 StGB) oder eines Schwangerschaftsabbruchs ohne Einhaltung bestimmter Voraussetzungen (Art. $120 \mathrm{StGB}$ ) verschont. Ursächlich für die Eröffnung einer Strafuntersuchung ist meist eine Strafanzeige entweder des eigenen Patienten oder eines Dritten, welcher z. B. Ansprüche gegen den Arzt durchzusetzen versucht.

\section{Strafuntersuchung - was heisst das?}

Eine Strafuntersuchung wird eröffnet, wenn ein konkreter Tatverdacht gegen die beschuldigte Person besteht. Federführend für die Strafuntersuchung ist die Staatsanwaltschaft (in einigen Kantonen Untersuchungsrichteramt genannt) und nicht, wie es der Laie häufig glaubt, die Anzeige erstattende Person. Ein Geschädigter hat gewisse Teilnahmerechte und kann Zivilansprüche geltend machen, für die Strafuntersuchung bleibt aber die Staatsanwaltschaft verantwortlich. Die Staatsanwaltschaft sichert die Beweise und untersucht, ob sich die angeschuldigte Person eines

\section{Enquêtes pénales à l'encontre de médecins: \\ comment se comporter?}

Toute ouverture d'une enquête pénale à l'encontre d'un médecin doit être prise au sérieux dès le début. Si la police ou le ministère public devaient procéder à l'improviste à une perquisition du cabinet, le médecin devrait immédiatement demander que tous les documents soient scellés, ou du moins les dossiers des patients, et faire preuve de la plus grande retenue dans ses affirmations. En adoptant dès le début la bonne stratégie, il devient possible d'influencer positivement la procédure.

strafbaren Verhaltens schuldig gemacht hat oder nicht. Je nach Ergebnis der Strafuntersuchung wird das Verfahren gegen den Angeschuldigten eingestellt oder es wird Anklage bei einem Gericht erhoben. In Fällen, in denen sich der Angeschuldigte geständig zeigt und die zu einer relativ geringen Strafe führen (im Kanton Zürich Freiheitsstrafe von höchstens 3 Monaten), kann das Verfahren mittels Strafbefehl, der einem Gerichtsurteil gleichkommt, abgeschlossen werden.

\section{Zwangsmassnahmen gegen den angeschuldigten Arzt}

Zu Beginn jeder Strafuntersuchung besteht das Bestreben, Beweismittel zu sichern. Beweismittel sind Aussagen des Angeschuldigten, von Zeugen, von Auskunftspersonen oder Sachverständigen, daneben aber auch Urkunden oder der Augenscheinsbeweis.

Zur Sicherung solcher Beweismittel kann die Staatsanwaltschaft Zwangsmassnahmen anordnen. Solche können eine Hausdurchsuchung, verbunden 
mit der Beschlagnahmung von Dokumenten, Computern oder anderen Gegenständen sein, daneben aber auch eine Telefonüberwachung oder gar Verhaftung des Angeschuldigten. In den Strafuntersuchungen gegen Ärzte dürfte die Hausdurchsuchung mit Beschlagnahmung von Dokumenten, insbesondere Patientendossiers, die häufigste Zwangsmassnahme sein.

\section{Hausdurchsuchung in der Arztpraxis - Verpflichtung zur Herausgabe von Patientendossiers?}

Steht z. B. die Richtigkeit eines Arztzeugnisses in Frage, und wird ein Arzt wegen des Vorwurfs eines falschen ärztlichen Zeugnisses bei der Polizei oder der Staatsanwaltschaft angezeigt, riskiert er eine für ihn völlig überraschende und selbstverständlich unangekündigte Hausdurchsuchung in seinen Praxisräumen. Diese wird häufig durch die Polizei vorgenommen, die - ausser bei zeitlicher Dringlichkeit - über einen Hausdurchsuchungsbefehl verfügen muss, den man sich zeigen lassen sollte. Grundsätzlich ist es selten sinnvoll, sich einer Hausdurchsuchung zu widersetzen, und es empfiehlt sich häufig sogar, der Behörde beim Auffinden von gesuchten Dokumenten behilflich zu sein, damit sich die Beschlagnahmung auf das Nötigste reduziert und die Durchsuchung nicht ausufert.

\section{Siegelung von Dokumenten}

\section{mit Geheimhaltungsinteressen}

Sind Dokumente von der Beschlagnahmung betroffen, die ein Geheimhaltungsinteresse bergen, wie z.B. die Krankenakte eines Patienten, so kann und soll die Siegelung dieser Dokumente verlangt werden. Eine Siegelung der Akten muss unmittelbar mit der Beschlagnahmung der Akten, also nicht erst später bei der eigentlichen Auswertung, verlangt werden. Wer dies verpasst, kann in aller Regel eine solche später nicht mehr nachholen, insbesondere nicht mit dem Argument, dass der Arzt, der von der Hausdurchsuchung betroffen war, nicht wusste, was eine Siegelung ist. Das Recht, die Siegelung zu verlangen, ist in der Regel auf dem Protokoll über die Hausdurchsuchung aufgedruckt, wenn auch meistens nur auf der Rückseite und kleingedruckt.

Eine Siegelung hat zur Folge, dass die beschlagnahmten Dokumente nicht einfach zu den Untersuchungsakten genommen und ausgewertet werden dürfen, sondern dass in einem gerichtlichen Verfahren darüber entschieden werden muss, ob die Papiere in die Akten einzubeziehen sind. Das Gericht prüft, ob ein hinreichender Tatverdacht besteht, und ob die Geheimnisinteressen gegenüber dem Untersuchungsinteresse zurückzutreten haben. Bis zur Bewilligung der Entsiegelung durch ein Gericht besteht ein Verwertungsverbot.

Eine Siegelung gibt dem Arzt die Möglichkeit, dass er zuerst gegenüber der Staatsanwaltschaft mündlich seine medizinischen Überlegungen dartun kann, bevor dieser sich als medizinischer Laie, gestützt auf für ihn oftmals nicht klar verständliche Schriftstücke, eine Meinung fasst. Auf diese Weise kann es gelingen, dass einvernehmlich mit der Staatsanwaltschaft mindestens ein Teil des Patientendossiers zurückgegeben und nicht zu den Strafakten genommen wird, womit einerseits das Geheimnisinteresse des Patienten teilweise gewahrt bleibt und man andererseits im Strafverfahren nicht zusätzliche Angriffsfläche bildet. Mit einer Siegelung der Dokumente gewinnt der betroffene Arzt zudem wertvolle Zeit, um seine Verteidigung zu organisieren und sich über seine Rechte und den gegen ihn bestehenden Tatverdacht zu informieren.

\section{Aussagen durch den Angeschuldigten}

Wer als Angeschuldigter in einer Strafuntersuchung steckt, wird regelmässig von der Polizei oder der Staatsanwaltschaft befragt. Hierbei werden die Angeschuldigten zwar auf ihr Recht aufmerksam gemacht, die Aussage verweigern zu können, doch erinnert diese Belehrung mehr an die Kriminalfälle in amerikanischen Filmen und wird kaum als reale Verteidigungsmöglichkeit wahrgenommen. Da es ein menschliches Bedürfnis ist, sich zu verteidigen, wenn man mit einem Vorwurf (evtl. sogar einem falschen) konfrontiert wird, sind die meisten beschuldigten Personen bereit, sofort und ausführlich Aussagen vor der Untersuchungsbehörde zu machen. Die wenigsten sind sich bewusst, dass sie damit Gefahr laufen, sich selbst eine Schlinge um den Hals zu legen, aus der es später vielleicht kein Entrinnen mehr gibt. Aus Sicht eines Strafverteidigers ist klar, dass Aussagen nur dann deponiert werden dürfen, wenn der Vorwurf präzise und klar formuliert wird, und selbst dann sind Aussagen kurz und knapp $\mathrm{zu}$ halten. Es ist Sache der Untersuchungsbehörde, eine Schuld des Angeschuldigten zu beweisen, und nicht, wie es der Laie oft verstehen will, Aufgabe des Beschuldigten, seine Unschuld darzutun. Eine Erklärung des eigenen Verhaltens einfach aufs Geratewohl, ohne das Ziel der Befragung zu kennen, ist vergleichbar mit der Einnahme von Medikamenten ohne Wissen darum, was einem fehlt. Es empfiehlt sich daher oft, sich professionell für seine eigene Verteidigung beraten zu lassen. Die weit verbreitete Auffassung, es könne einem ja nichts passieren, wenn man sich nichts zuschulden habe kommen lassen, entpuppt sich immer wieder als folgenschwerer Irrtum.

Bei Aussagen gegenüber der Polizei und der Staatsanwaltschaft ist stets zu beachten, dass diese die Taktik verfolgen, mit der Befragung den Tatverdacht zu erhärten. Beantwortet man die ersten Fragen des Staatsanwaltes weitschweifig, birgt dies die Gefahr, dass man schliesslich erklären muss, dass man dies und jenes anders gemeint habe, und sich dadurch in Widersprüche verwickelt. Das später urteilende Gericht wird dieses widersprüchliche Aussageverhalten möglicherweise als unglaubwürdig werten, und der ursprünglich gut gemeinte Erklärungsversuch des Angeschuldigten kehrt wie ein Bumerang mit einem Schuldspruch zurück. 